\title{
The Generalization of Dirac's Theorem for Hypergraphs
}

\author{
Endre Szemerédi ${ }^{1}$, Andrzej Ruciński ${ }^{2, \star}$, and Vojtěch Rödl ${ }^{3, \star \star}$ \\ ${ }^{1}$ Rutgers University, New Brunswick \\ szemered@cs.rutgers.edu \\ ${ }^{2}$ A. Mickiewicz University, Poznań, Poland \\ rucinski@amu.edu.pl \\ ${ }^{3}$ Emory University, Atlanta, GA \\ rodl@mathcs . emory . edu
}

\section{Introduction and Main Result}

A substantial amount of research in graph theory continues to concentrate on the existence of hamiltonian cycles and perfect matchings. A classic theorem of Dirac states that a sufficient condition for an $n$-vertex graph to be hamiltonian, and thus, for $n$ even, to have a perfect matching, is that the minimum degree is at least $n / 2$. Moreover, there are obvious counterexamples showing that this is best possible.

The study of hamiltonian cycles in hypergraphs was initiated in [1] where, however, a different definition than the one considered here was introduced. Given an integer $k \geq 2$, a $k$-uniform hypergraph is a hypergraph (a set system) where every edge (set) is of size $k$.

By a cycle we mean a $k$-uniform hypergraph whose vertices can be ordered cyclically $v_{1}, \ldots, v_{l}$ in such a way that for each $i=1, \ldots, l$, the set $\left\{v_{i}, v_{i+1}, \ldots, v_{i+k-1}\right\}$ is an edge, where for $h>l$ we set $v_{h}=v_{h-l}$. A hamiltonian cycle in a $k$-uniform hypergraph $H$ is a spanning cycle in $H$, that is, a sub-hypergraph of $H$ which is a cycle and contains all vertices of $H$. A $k$-uniform hypergraph containing a hamiltonian cycle is called hamiltonian.

This notion and its generalizations have a potential to be applicable in many contexts which still need to be explored. An application in the relational database theory can be found in [2]. As observed in [5], the square of a (graph) hamiltonian cycle naturally coincides with a hamiltonian cycle in a hypergraph built on top of the triangles of the graph. More precisely, given a graph $G$, let $\operatorname{Tr}(G)$ be the set of triangles in $G$. Define a hypergraph $H^{\operatorname{Tr}}(G)=(V(G), \operatorname{Tr}(G))$. Then there is a one-to-one correspondence between hamiltonian cycles in $H^{T r}(G)$ and the squares of hamiltonian cycles in $G$. For results about the existence of squares of hamiltonian cycles see, e.g., [6].

As another potential application consider a seriously ill patient taking 24 different pills on a daily basis, one at a time every hour. Certain combinations

\footnotetext{
* Research supported by KBN grant 2 P03A 015 23. Part of research performed at Emory University, Atlanta.

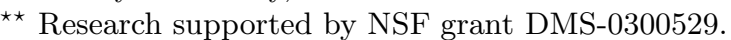


of three pills can be deadly if taken within 2.5 hour. Let $D$ be the set of deadly triplets of pills. Then any safe schedule corresponds to a hamiltonian cycle in the hypergraph which is precisely the complement of $D$.

A natural extension of Dirac's theorem to $k$-graphs, $k \geq 2$, has been conjectured in [5], where as a sufficient condition one demands that every $(k-1)$ element set of vertices is contained in at least $\lfloor n / 2\rfloor$ edges. The following construction of a $k$-uniform hypergraph $H_{0}$, also from [5], shows that the above conjecture, if true, is nearly best possible (best possible for $k=3$ ).

Let $V=V^{\prime} \cup\{v\},|V|=n$. Split $V^{\prime}=X \cup Y$, where, $|X|=\left\lfloor\frac{n-1}{2}\right\rfloor$ and $|Y|=$ $\left\lceil\frac{n-1}{2}\right\rceil$. The edges of $H_{0}$ are all $k$-element subsets $S$ of $V$ such that $|X \cap S| \neq\left\lfloor\frac{k}{2}\right\rfloor$ or $v \in S$. It is shown in [5] that $H_{0}$ is not hamiltonian, while every $(k-1)$-element set of vertices belongs to at least $\left\lfloor\frac{n-k+1}{2}\right\rfloor$ edges.

In [9] we proved an approximate version of the conjecture from [5] for $k=3$, and in [11] we give a generalization of that result to $k$-uniform hypergraphs for arbitrary $k$.

Theorem 1 ([11]). Let $k \geq 3$ and $\gamma>0$. Then, for sufficiently large $n$, every $k$-uniform hypergraph on $n$-vertices such that each $(k-1)$-element set of vertices is contained in at least $(1 / 2+\gamma) n$ edges is hamiltonian.

\section{The Idea of Proof}

The idea of the proof is as follows. As a preliminary step, we find in $H$ a powerful path $A$, called absorbing which has the property that every not too large subset of vertices can be "absorbed" by that path. We also put aside a small subset of vertices $R$ which preserves the degree properties of the entire hypergraph.

On the sub-hypergraph $H^{\prime}=H-(A \cup R)$ we find a collection of long, disjoint paths which cover almost all vertices of $H^{\prime}$. Then, using $R$ we "glue" them and the absorbing path $A$ together to form a long cycle in $H$. In the final step, the vertices which are not yet on the cycle are absorbed by $A$ to form a hamiltonian cycle in $H$.

The main tool allowing to cover almost all vertices by disjoint paths is a generalization of the regularity lemma from [12].

Given a $k$-uniform hypergraph $H$ and $k$ non-empty, disjoint subsets $A_{i} \subset$ $V(H), i=1, \ldots, k$, we define $e_{H}\left(A_{1}, \ldots, A_{k}\right)$ to be the number of edges in $H$ with one vertex in each $A_{i}$, and the density of $H$ with respect to $\left(A_{1}, \ldots, A_{k}\right)$ as

$$
d_{H}\left(A_{1}, \ldots, A_{k}\right)=\frac{e_{H}\left(A_{1}, \ldots, A_{k}\right)}{\left|A_{1}\right| \cdots\left|A_{k}\right|} .
$$

A $k$-uniform hypergraph $H$ is $k$-partite if there is a partition $V(H)=V_{1} \cup$ $\cdots \cup V_{k}$ such that every edge of $H$ intersects each set $V_{i}$ in precisely one vertex. For a $k$-uniform, $k$-partite hypergraph $H$, we will write $d_{H}$ for $d_{H}\left(V_{1}, \ldots, V_{k}\right)$ and call it the density of $H$.

We say that a $k$-uniform, $k$-partite hypergraph $H$ is $\epsilon$-regular if for all $A_{i} \subseteq V_{i}$ with $\left|A_{i}\right| \geq \epsilon\left|V_{i}\right|, i=1, \ldots, k$, we have

$$
\left|d_{H}\left(A_{1}, \ldots, A_{k}\right)-d_{H}\right| \leq \epsilon .
$$


The following result, called weak regularity lemma as opposed to the stronger result in 4], is a straightforward generalization of the graph regularity lemma from $[12$.

Lemma 1 (Weak regularity lemma for hypergraphs). For all $k \geq 2$, every $\epsilon>0$ and every integer $t_{0}$ there exist $T_{0}$ and $n_{0}$ such that the following holds. For every $k$-uniform hypergraph $H$ on $n>n_{0}$ vertices there is, for some $t_{0} \leq t \leq T_{0}$, a partition $V(H)=V_{1} \cup \cdots \cup V_{t}$ such that $\left|V_{1}\right| \leq\left|V_{2}\right| \leq \cdots \leq\left|V_{t}\right| \leq\left|V_{1}\right|+1$ and for all but at most $\epsilon t^{k}$ sets of partition classes $\left\{V_{i_{1}}, \ldots, V_{i_{k}}\right\}$, the induced $k$-uniform, $k$-partite sub-hypergraph $H\left[V_{i_{1}}, \ldots, V_{i_{k}}\right]$ of $H$ is $\epsilon$-regular.

The above regularity lemma, combined with the fact that every dense $\epsilon$ regular hypergraph contains an almost perfect path-cover, yields an almost perfect path-cover of the entire hypergraph $H$.

\section{Results for Matchings}

A perfect matching in a $k$-uniform hypergraph on $n$ vertices, $n$ divisible by $k$, is a set of $n / k$ disjoint edges. Clearly, every hamiltonian, $k$-uniform hypergraph with the number of vertices $n$ divisible by $k$ contains a perfect matching.

Given a $k$-uniform hypergraph $H$ and a $(k-1)$-tuple of vertices $v_{1}, \ldots, v_{k-1}$, we denote by $N_{H}\left(v_{1}, \ldots, v_{k-1}\right)$ the set of vertices $v \in V(H)$ such that $\left\{v_{1}, \ldots\right.$, $\left.v_{k-1}, v\right\} \in H$. Let $\delta_{k-1}(H)=\delta_{k-1}$ be the minimum of $\left|N_{H}\left(v_{1}, \ldots, v_{k-1}\right)\right|$ over all $(k-1)$-tuples of vertices in $H$.

For all integer $k \geq 2$ and $n$ divisible by $k$, denote by $t_{k}(n)$ the smallest integer $t$ such that every $k$-uniform hypergraph on $n$ vertices and with $\delta_{k-1} \geq t$ contains a perfect matching.

For $k=2$, that is, in the case of graphs, we have $t_{2}(n)=n / 2$. Indeed, the lower bound is delivered by the complete bipartite graph $K_{n / 2-1, n / 2+1}$, while the upper bound is a trivial corollary of Dirac's condition [3. for the existence of Hamilton cycles.

In [10] we study $t_{k}$ for $k \geq 3$. As a by-product of our result about hamiltonian cycles in [1] (see Theorem 2 above), it follows that $t_{k}(n)=n / 2+o(n)$. Kühn and Osthus proved in 7 that

$$
\frac{n}{2}-k+1 \leq t_{k}(n) \leq \frac{n}{2}+3 k^{2} \sqrt{n \log n} .
$$

The lower bound follows by a simple construction, which, in fact, for $k$ odd yields $t_{k}(n) \geq n / 2-k+2$. For instance, when $k=3$ and $n / 2$ is an odd integer, split the vertex set into sets $A$ and $B$ of size $n / 2$ each, and take as edges all triples of vertices which are either disjoint from $A$ or intersect $A$ in precisely two elements.

In [10] we improve the upper bound from [7].

Theorem 2. For every integer $k \geq 3$ there exists a constant $C>0$ such that for sufficiently large $n$,

$$
t_{k}(n) \leq \frac{n}{2}+C \log n
$$


It is very likely that the true value of $t_{k}(n)$ is yet closer to $n / 2$. Indeed, in [5] it is conjectured that $\delta_{k-1} \geq n / 2$ is sufficient for the existence of a Hamilton cycle, and thus, when $n$ is divisible by $k$, the existence of a perfect matching. Based on this conjecture and on the above mentioned construction from [7], we believe that $t_{k}(n)=n / 2-O(1)$. In fact, for $k=3$, we conjecture that $t_{3}(n)=\lceil n / 2\rceil-1$.

Our belief that $t_{k}(n)=n / 2-O(1)$ is supported by some partial results. For example, we are able to show that the threshold function $t_{k}(n)$ has a stability property, in the sense that hypergraphs that are "away" from the "extreme case" $H_{0}$, described in Section 1, contain a perfect matching even when $\delta_{k-1}$ is smaller than but not too far from $n / 2$.

Interestingly, if we were satisfied with only a partial matching, covering all but a constant number of vertices, then this is guaranteed already with $n / 2+o(n)$ replaced by $n / k$, that is, when $\delta_{k-1} \geq n / k$.

We have also another related result, about the existence of a fractional perfect matching, which is a simple consequence of Farkas' Lemma (see, e.g., [8]). A fractional perfect matching in a $k$-uniform hypergraph $H=(V, E)$ is a function $w: E \rightarrow[0,1]$ such that for each $v \in V$ we have

$$
\sum_{e \ni v} w(e)=1
$$

In particular, it follows from our result that if $\delta_{k-1}(H) \geq n / k$ then $H$ has a fractional perfect matching, so, again, the threshold is much lower than that for perfect matchings.

\section{References}

1. J. C. Bermond et al., Hypergraphes hamiltoniens, Prob. Comb. Theorie Graph Orsay 260 (1976) 39-43.

2. J. Demetrovics, G. O. H. Katona and A. Sali, Design type problems motivated by database theory, Journal of Statistical Planning and Inference 72 (1998) 149-164.

3. G. A. Dirac, Some theorems for abstract graphs, Proc. London Math. Soc. (3) 2 (1952) 69-81.

4. P. Frankl and V. Rödl, Extremal problems on set systems, Random Struct. Algorithms 20, no. 2, (2002) 131-164.

5. Gyula Y. Katona and H. A. Kierstead, Hamiltonian chains in hypergraphs, $J$. Graph Theory 30 (1999) 205-212.

6. J. Komlós, G. N. Sárközy and E. Szemerédi, On the Pósa-Seymour conjecture, J. Graph Theory 29 (1998) 167-176.

7. D. Kuhn and D. Osthus, Matchings in hypergraphs of large minimum degree, submited.

8. L. Lovász \& M.D. Plummer, Matching theory. North-Holland Mathematics Studies 121, Annals of Discrete Mathematics 29, North-Holland Publishing Co., Amsterdam; Akadémiai Kiadó, Budapest, 1986

9. V. Rödl, A. Ruciński and E. Szemerédi, A Dirac-type theorem for 3-uniform hypergraphs, Combinatorics, Probability and Computing, to appear.

10. V. Rödl, A. Ruciński and E. Szemerédi, Perfect matchings in uniform hypergraphs with large minimum degree, submitted. 
11. V. Rödl, A. Ruciński and E. Szemerédi, An approximative Dirac-type theorem for $k$-uniform hypergraphs, submitted.

12. E. Szemerédi, Regular partitions of graphs. Problemes combinatoires et theorie des graphes (Colloq. Internat. CNRS, Univ. Orsay, Orsay, 1976), pp. 399-401, Colloq. Internat. CNRS, 260, CNRS, Paris, 1978. 